\title{
Article
}

\section{Increased Serum Levels of S100A4 and S100A15 in Individuals Suffering from Hidradenitis Suppurativa}

\author{
Aleksandra Batycka-Baran *, Łukasz Matusiak, Danuta Nowicka-Suszko, Jacek C. Szepietowski *D \\ and Wojciech Baran (D)
}

Citation: Batycka-Baran, A.;

Matusiak, Ł.; Nowicka-Suszko, D.; Szepietowski, J.C.; Baran, W. Increased Serum Levels of S100A4 and S100A15 in Individuals Suffering from Hidradenitis Suppurativa. J. Clin. Med. 2021, 10, 5320. https:// doi.org/10.3390/jcm10225320

Academic Editor: Dennis Paul Orgill

Received: 9 October 2021

Accepted: 10 November 2021

Published: 15 November 2021

Publisher's Note: MDPI stays neutral with regard to jurisdictional claims in published maps and institutional affiliations.

Copyright: (c) 2021 by the authors. Licensee MDPI, Basel, Switzerland. This article is an open access article distributed under the terms and conditions of the Creative Commons Attribution (CC BY) license (https:// creativecommons.org/licenses/by/ $4.0 /)$.
Department of Dermatology, Venereology and Allergology, Wroclaw Medical University, 50-368 Wroclaw, Poland; lukasz.matusiak@umed.wroc.pl (Ł.M.); danuta.nowicka-suszko@umed.wroc.pl (D.N.-S.); wojciech.baran@umed.wroc.pl (W.B.)

* Correspondence: ola.batycka@interia.pl (A.B.-B.); jacek.szepietowski@umed.wroc.pl (J.C.S.); Tel.: +48-71-327-0941 (A.B.-B. \& J.C.S.); Fax: +48-71-327-0942 (A.B.-B. \& J.C.S.)

\begin{abstract}
Hidradenitis suppurativa (HS) is a chronic inflammatory skin disease. Recently, some S100 proteins have been suggested to play an important role in the pathogenesis of chronic immunemediated inflammatory diseases and they may constitute valuable biomarkers for these diseases' diagnosis and monitoring. The objective of the current study was to investigate, for the first time, serum levels of S100A4 and S100A15 in individuals suffering from HS. Furthermore, we assessed the associations between S100A4 and S100A15 serum levels and the severity of disease, CRP serum concentration and some demographic and clinical data. Serum levels of S100A4 and S100A15 were evaluated with the commercially available ELISA kit according to the manufacturer's instructions. The serum level of S100A4 in individuals with HS was significantly elevated as compared to controls, with the highest level found in the individuals in Hurley stage II. The S100A15 serum level was positively correlated with the CRP concentration and was associated with the severity of the disease. The serum level of S100A15 in the individuals in Hurley stage III was significantly elevated compared to that of the controls and the individuals with HS in Hurley stages I and II. S100A4 and S100A15 may be considered as new serum biomarkers for the monitoring of HS progression, and they may play a role in the pathogenesis of HS by promoting inflammatory process and fibrosis.
\end{abstract}

Keywords: S100A4; S100A15; hidradenitis suppurativa

\section{Introduction}

Hidradenitis suppurativa (HS)/acne inversa is a chronic inflammatory and suppurative skin disease that affects intertriginous areas of the body, such as the axillary, inguinal and anogenital regions. It clinically manifests as tender nodules and abscesses; additionally, fistulas, fibrosis, scarring and dermal contractures may appear as the disease progresses [1-3]. The pathogenesis of HS is still not completely elucidated and requires further investigation. Recently, the important role of aberrant innate immune responses in epidermal abnormalities has been highlighted [3-5]. Some recent studies suggest that a defect in antimicrobial response may be present in individuals with HS, that may result from a deficiency of interleukin (IL)-20, IL-22 or the ineffective antimicrobial activity of IL-26 [6,7]. Further, HS is currently considered to be a chronic systemic inflammatory disease that is associated with metabolic and cardiovascular comorbidities [1,8-10]. The dignosis of HS is still usually made clinically. There are several scoring systems for the assessment of the disease's severity, mainly based on clinical criteria. However, all of them have some limitations in daily practice. The Hurley classification system was first suggested in 1989, and it is still widely used [1,2]. In HS, the course and progression of the disease are difficult to predict. Currently, there is a lack of suitable plasma or serum biomarkers that could facilitate differentiation between stages and inform prediction of the progression of the disease [1,11]. S100 proteins belong to a family of low-molecular-weight 
(9-13 kDa), calcium-binding proteins that are involved in extra- and intra-cellular signaling and the regulation of cell proliferation and differentiation. Recently, some S100 proteins have been suggested to play an important role in the pathogenesis of chronic immunemediated inflammatory diseases and, therefore, it is thought that they may constitute valuable biomarkers for use in diagnosis and monitoring [11-13]. Similarly, calprotectin (S100A8/S100A9) has been suggested to be a predictive biomarker of adalimumab response in HS patients [14]. As members of the S100 protein family, S100A4 and S100A15 are encoded within a frequently rearranged gene cluster, namely the epidermal differentiation complex on chromosome 1q21 [13,15,16]. S100A4 (metastasin, a fibroblast-specific protein) is well known to be involved in cancer progression and metastasis. However, S100A4 is also expressed in non-tumor cells (e.g., fibroblasts, activated lymphocytes, neutrophils and macrophages) and is involved in various non-malignant pathophysiologies, such as immune response, inflammation and angiogenesis. Furthermore, it promotes tissue fibrosis and is considered to be a specific fibroblast marker. S100A4 is involved in the pathogenesis of kidney and pulmonary fibrosis, as well as psoriasis, systemic sclerosis and hypertrophic scarring [16-21]. S100A15 was first identified from its overexpression in 'koebnerized' psoriatic skin; therefore, it was named koebnerisin [15]. Recently, an overexpression of S100A15 was found in the lesional and perilesional skin of individuals with HS compared to the skin of healthy controls [22]. S100A15 is an antimicrobial protein that reduces the survival rates of Escherichia coli, Staphylococcus aureus and Pseudomonas aeruginosa. It regulates keratinocyte proliferation and differentiation. Further, S100A15 has been identified as an endogenous danger-associated molecular pattern (DAMPS) with proinflammatory properties. S100A15 has been shown to prime keratinocytes for the enhanced production of proinflammatory cytokines, including TNF- $\alpha$, IL- 6 and IL-8, and it has been found to act as a chemotactic factor for neutrophils and monocytes/macrophages $[15,23,24]$. The objective of the current study was to investigate, for the first time, serum levels of S100A4 and S100A15 in individuals suffering from HS. Furthermore, we assessed the associations of S100A4 and S100A15 serum levels with the severity of HS, CRP serum concentration and some demographic and clinical data.

\section{Materials and Methods}

The study group consisted of 61 individuals suffering from HS (31 females/30 males) and 30 healthy controls (16 females/14 males) matched by age and sex. The diagnoses of HS were based on well-established clinical criteria (3). All patients with HS had active disease with the presence of inflammatory lesions (abscesses and nodules). HS patients were biologically naive and had not received any local or systemic anti-inflammatory therapy (e.g., antibiotics or retinoids) for at least 8 weeks prior to the initiation of the study. The age of subjects ranged from 19 to 67 years (mean $\pm \mathrm{SD}=38.5 \pm 10.9$ ). The mean duration of the disease was $8.7 \pm 7.4$ years, ranging from 2 to 27 years. The level of severity of disease was assessed according to the Hurley staging system; 19 individuals scored at stage I (31.1\% of the HS cohort), 28 individuals scored at stage II (45.9\%) and 14 individuals scored at stage III( $22.9 \%)$. The mean BMI score for the HS cohort was $28.9 \pm 4.9$, with an obesity percentage of $42.6 \%$ (where obesity is defined as BMI $\geq 30$, as per the WHO). The percentage of smokers among the HS cohort was $63.9 \%$. The control group consisted of healthy subjects. The mean age was $39.3 \pm 10.3$ years and the mean BMI was $25.6 \pm 5.8$, with an obesity percentage of $20 \%$. The percentage of smokers among the control cohort was $16.6 \%$. Exclusion criteria included other skin diseases, chronic infection, inflammatory bowel disease, chronic kidney, liver or heart diseases and malignancies (Table 1).

From each subject, $5 \mathrm{~mL}$ of peripheral blood was taken. The assessment of serum concentrations of S100A4 and S100A15 was performed using a commercially available ELISA kit, according to the manufacturer's instructions (MyBiosource Inc., San Diego, CA, USA). 
Table 1. Characteristics of population.

\begin{tabular}{|c|c|c|c|c|c|c|}
\hline & & \multicolumn{3}{|c|}{ Patients } & Controls & $p$ Value \\
\hline \multicolumn{2}{|l|}{ Gender F/M } & \multicolumn{3}{|c|}{$31 / 30$} & $16 / 14$ & $p>0.05$ \\
\hline \multicolumn{2}{|l|}{ Age (years) } & \multicolumn{3}{|c|}{$38.5 \pm 10.9$} & $39.3 \pm 10.3$ & $p>0.05$ \\
\hline \multicolumn{2}{|l|}{$\mathrm{BMI}\left(\mathrm{kg} / \mathrm{m}^{2}\right)$} & \multicolumn{3}{|c|}{$28.9 \pm 4.9$} & $25.6 \pm 4.2$ & $* p<0.05$ \\
\hline \multicolumn{2}{|c|}{ Disease duration (years) } & \multicolumn{3}{|c|}{$8.7 \pm 7.4$} & \multicolumn{2}{|c|}{$\mathrm{N} / \mathrm{A}$} \\
\hline \multicolumn{2}{|c|}{$\mathrm{CRP}(\mathrm{mg} / \mathrm{L})$} & \multicolumn{3}{|c|}{$17.4 \pm 25.2$} & $2.7 \pm 2.0$ & $* p<0.05$ \\
\hline \multicolumn{2}{|c|}{ Smokers/non-smokers } & \multicolumn{3}{|c|}{$39 / 22$} & $5 / 25$ & $* p<0.05$ \\
\hline \multirow{4}{*}{ Hurley staging } & & Females & Males & Total & \multirow{4}{*}{\multicolumn{2}{|c|}{$\mathrm{N} / \mathrm{A}$}} \\
\hline & Hurley I & 12 & 7 & 19 & & \\
\hline & Hurley II & 15 & 13 & 28 & & \\
\hline & Hurley III & 4 & 10 & 14 & & \\
\hline
\end{tabular}

* $p<0.05$ statistically significant.

The assessment of serum C-reactive protein (CRP) was performed using a turbidimetric assay on the Architect ci4100 analyzer (Abbott Diagnostics, Lake Forest, IL, USA). Informed consent was obtained from all participants. The study was conducted according to the Declaration of Helsinki and approved by the local Bioethical Committee.

\section{Statistical Analysis}

The statistical analyses were performed using Statistica (version 13.1, StatSoft, Tulsa, OK, USA) and GraphPad Prism version 5.0. (La Jolla, CA, USA). The mean and standard deviations were calculated. Shapiro-Wilk and Kolmogorov-Smirnov normality tests were used to analyze whether variables were of normal or abnormal distribution. The significance of the differences between variables was determined using the Mann-Whitney U test, Pearson's $\chi^{2}$ test, chi-square with Yates' correction test and Fisher's exact test and the Kruskal-Wallis test. Relationships between continuous variables of interest were assessed using Spearman's rank correlation coefficient, and receiver operating characteristics (ROC) curve analysis was performed. Based on the ROC curve analysis, the area under the curve (AUC) and the optimal cut-off value were obtained. The optimal cut-off value was obtained from the Youden index (maximum (sensitivity + specificity -1$)$ ), i.e., as a point with the highest sensitivity and specificity. A $p$-value $<0.05$ was considered to be statistically significant.

\section{Results}

The serum concentration of S100A4 in the individuals suffering from HS was significantly elevated when compared to those in the controls $(31.57 \mathrm{pg} / \mathrm{mL} \pm 24.38$ vs. $22.51 \mathrm{pg} / \mathrm{mL} \pm 23.30, p=0.022)$ (Figure 1a).

There was a statistically significant difference in the S100A4 serum concentrations between the three Hurley stages $(p=0.03)$, with the highest concentration of S1004 being found in patients in Hurley stage II (Figure 1b, Table 2).

Table 2. Serum levels of S100A4, S100A15 and C-reactive protein (CRP) in healthy controls and patients with hidradenitis suppurativa, according to Hurley staging system.

\begin{tabular}{|c|c|c|c|c|}
\hline & $\mathbf{N}$ & $\begin{array}{c}\text { S100A4 }(\mathrm{pg} / \mathrm{mL}) \\
\text { Mean } \pm \text { SD }\end{array}$ & $\begin{array}{l}\mathrm{S} 100 \mathrm{~A} 15(\mathrm{pg} / \mathrm{mL}) \\
\text { Mean } \pm \mathrm{SD}\end{array}$ & $\begin{array}{l}\text { CRP }(\mathrm{mg} / \mathrm{L}) \\
\text { Mean } \pm \mathrm{SD}\end{array}$ \\
\hline Controls & 30 & $22.5 \pm 23.3$ & $153.9 \pm 134.0$ & $2.7 \pm 2.0$ \\
\hline Hurley I & 19 & $22.7 \pm 22.6$ & $50.8 \pm 30.9$ & $3.9 \pm 4.4$ \\
\hline Hurley II & 28 & $37.6 \pm 24.5$ & $151.5 \pm 115.7$ & $9.6 \pm 10.1$ \\
\hline Hurley III & 14 & $35.3 \pm 24.9$ & $317.1 \pm 101.0$ & $43.1 \pm 35.2$ \\
\hline
\end{tabular}




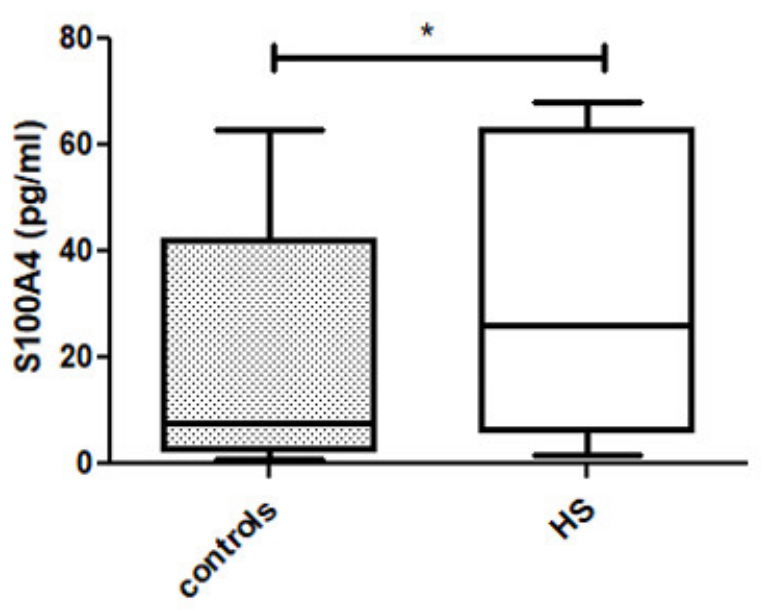

(a)

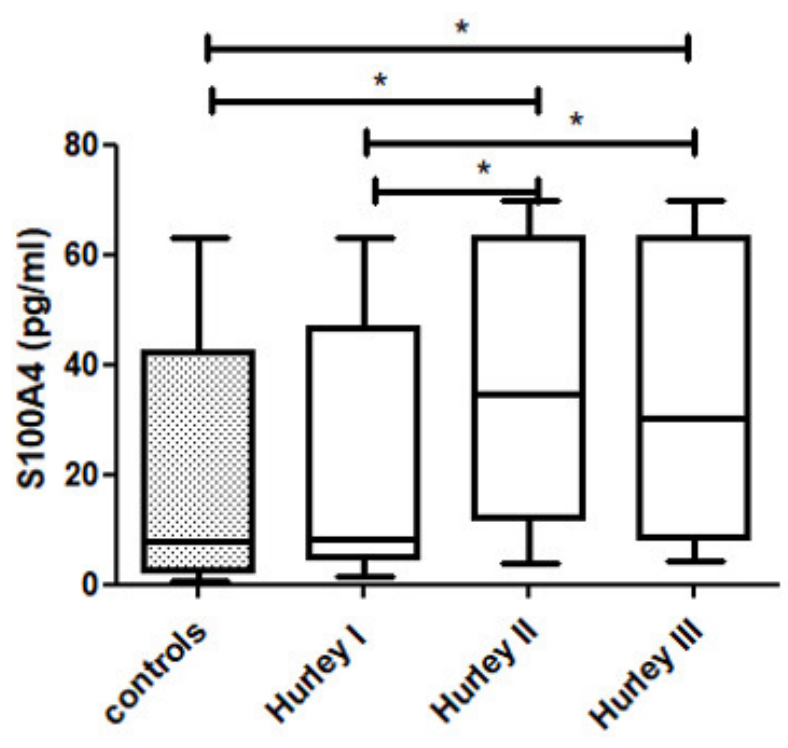

(b)

Figure 1. (a). The serum concentration of S100A4 in individuals suffering from HS was significantly elevated as compared to controls $(p=0.022)$. (b). There was statistically significant difference in S100A4 serum concentrations between Hurley stages $(p=0.03)$, with the highest concentration of S1004 in patients in Hurley II stage. The serum concentrations of S100A4 in patients in Hurley II stage was significantly elevated as compared to controls and individuals with HS in Hurley I stage ( $p=0.005, p=0.012$, respectively). The serum concentrations of S100A4 in patients in Hurley III stage was significantly elevated as compared to controls and individuals with HS in Hurley I stage ( $p=0.039, p=0.049$, respectively). ${ }^{*}$ is statistically significant result $p<0.05$.

The serum concentration of S100A4 in the patients in Hurley stage II was significantly elevated compared to that of the controls and the individuals with HS in Hurley stage I ( $p=0.005$ and $p=0.012$, respectively) (Figure $1 b$ ). Similarly, the serum concentrations of S100A4 in the patients with HS in Hurley stage III were significantly elevated as compared to those of the controls and the individuals with HS in Hurley stage I ( $p=0.039$ and $p=0.049$, respectively) (Figure 1b). There was no significant difference in the serum concentrations of S100A4 between the individuals with HS in Hurley stages II and III (Figure 1b). We did not find any significant correlations between the serum concentrations of S100A4 and CRP, BMI, smoking habit or other demographic and clinical data.

The ROC analysis of the S100A4 serum concentrations in the individuals with HS in Hurley stage II and Hurley stage III, as compared to the controls, showed an area under the curve (AUC) of 0.7 (95\%CI for AUC: $0.58-0.83)(p=0.0014)$. The optimal cut-off value was set at $6.02 \mathrm{pg} / \mathrm{mL}$ (Figure 2a).

The ROC analysis of the serum concentrations of S100A4 in the individuals with HS in Hurley stage II and Hurley stage III, as compared to the individuals with HS in Hurley stage I, showed an AUC of 0.72. (95\%CI for AUC: $0.58-0.86)(p=0.0022)$. The optimal cut-off value was set at $6.16 \mathrm{pg} / \mathrm{mL}$ (Figure $2 \mathrm{~b}$ ).

There was no significant difference in the serum concentration of S100A15 between the whole group of patients suffering from HS $(156.1 \pm 133.8 \mathrm{pg} / \mathrm{mL})$ and the control group $(153.9 \pm 134.8 \mathrm{pg} / \mathrm{mL})(p>0.05)$ (Figure 3a). The S100A15 serum concentration was associated with the severity of disease as classified according to the Hurley staging system; there was a statistically significant difference in S100A15 serum concentrations between the Hurley stages $(p<0.0001)$, with the highest concentration of S10015 being found in the patients in Hurley stage III (Figure 3b, Table 2). The serum concentration of S100A15 in the patients in Hurley stage III was significantly elevated compared to those in the controls $(p=0.0013)$ (Figure 3b). 


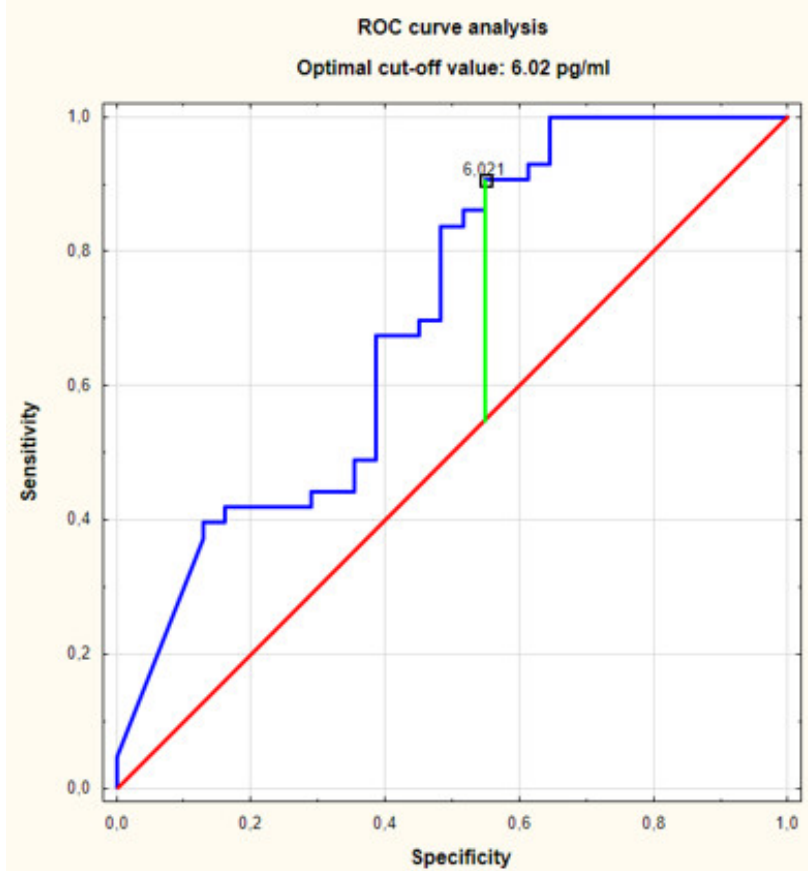

(a)

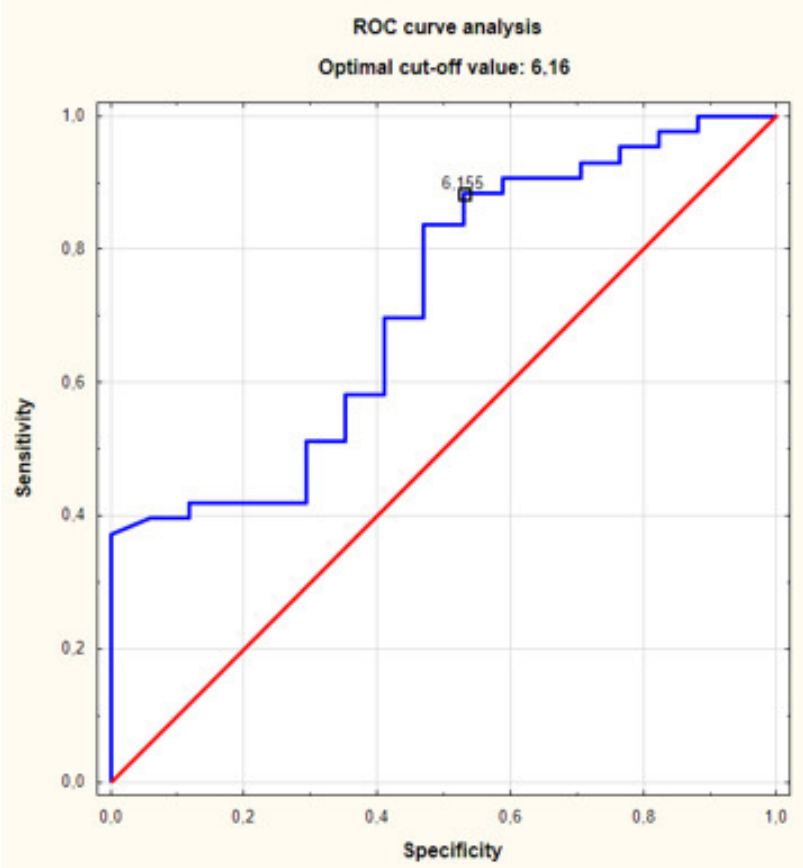

(b)

Figure 2. (a). ROC curve analysis of relationship between serum concentrations of S100A4 in individuals with HS in stage Hurley II + III and controls. Receiver operating characteristics (ROC) curves analysis showed area under the curve (AUC) of 0.7. (95\%CI for AUC: 0.58-0.83) ( $p=0.0014)$. (b). ROC curve analysis of relationship between serum concentrations of S100A4 in individuals with HS Hurley II + III and Hurley I. Receiver operating characteristics (ROC) curves analysis showed area under the curve (AUC) of 0.72 (95\%CI for AUC: $0.58-0.86)(p=0.0022)$.

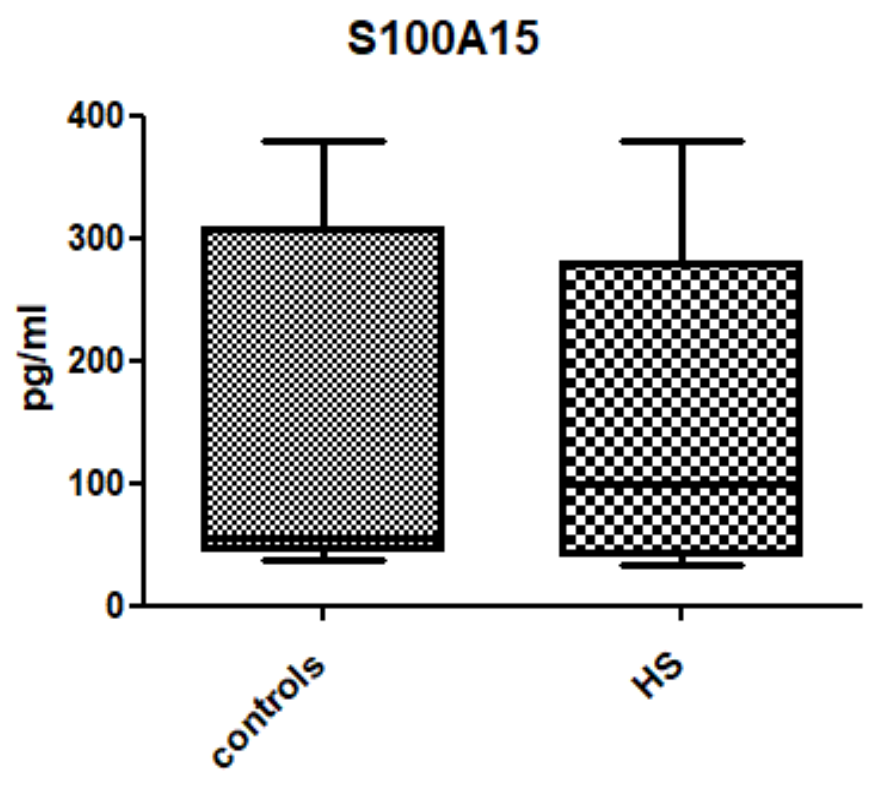

(a)

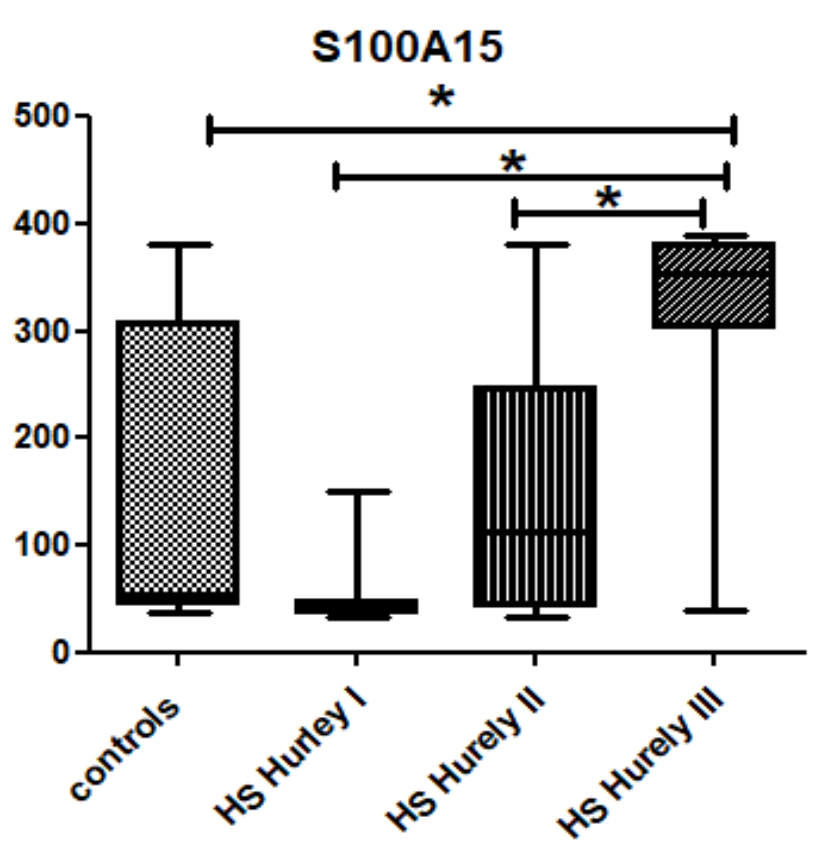

(b)

Figure 3. (a). There was no significant difference in serum concentrations of S100A15 between patients suffering from HS and controls $(p>0.05)$. (b). There was statistically significant difference in S100A15 serum concentrations between Hurley stages $(p<0.0001)$. The serum concentrations of S100A15 in patients in Hurley III stage were significantly elevated as compared to controls, individuals with HS in Hurley I stage and individuals with HS in Hurley II stage ( $p=0.0013$, $p<0.0001$ and $p=0.0004$ respectively). ${ }^{*}$ is statistically significant result $p<0.05$. 
The ROC curve analysis showed an AUC of 0.81 (95\%CI for AUC: $0.66-0.97)(p=0.0001)$. The optimal cut-off value was set at $312.85 \mathrm{pg} / \mathrm{mL}$ (Figure $4 \mathrm{a}$ ).

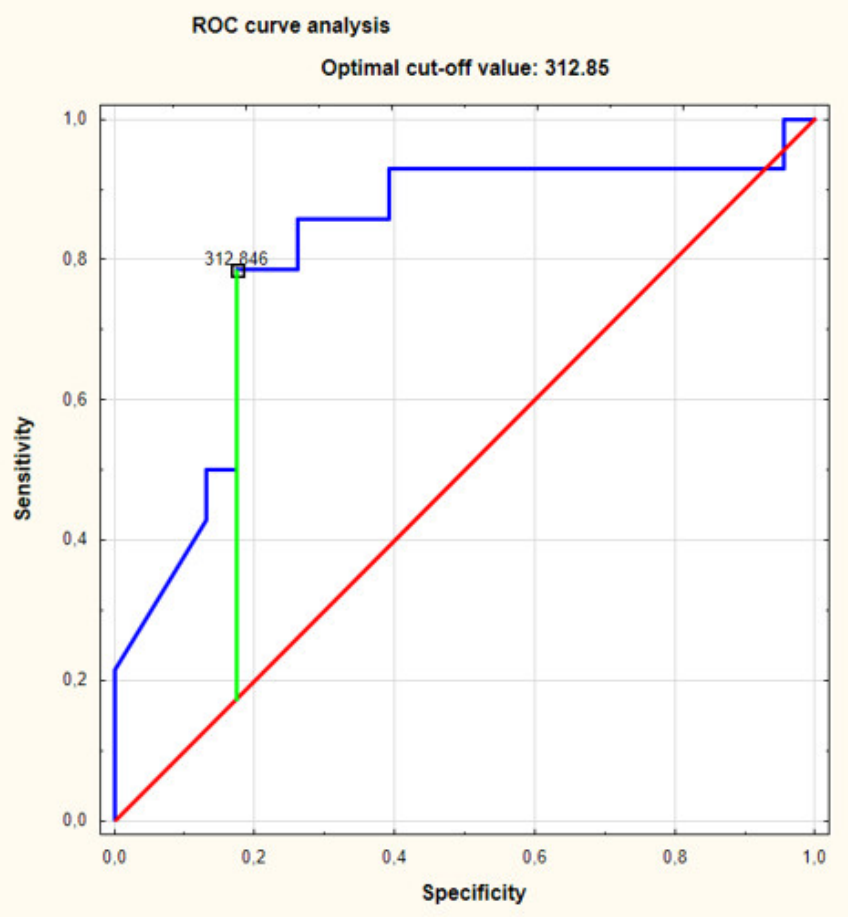

(a)

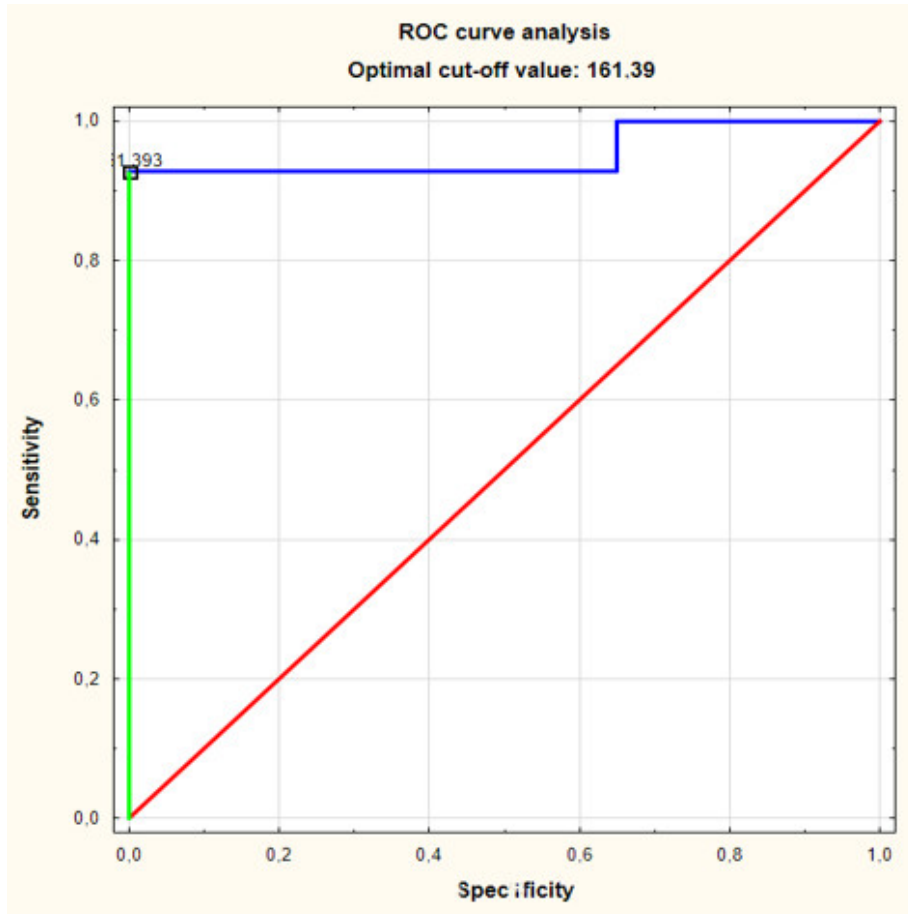

(b)

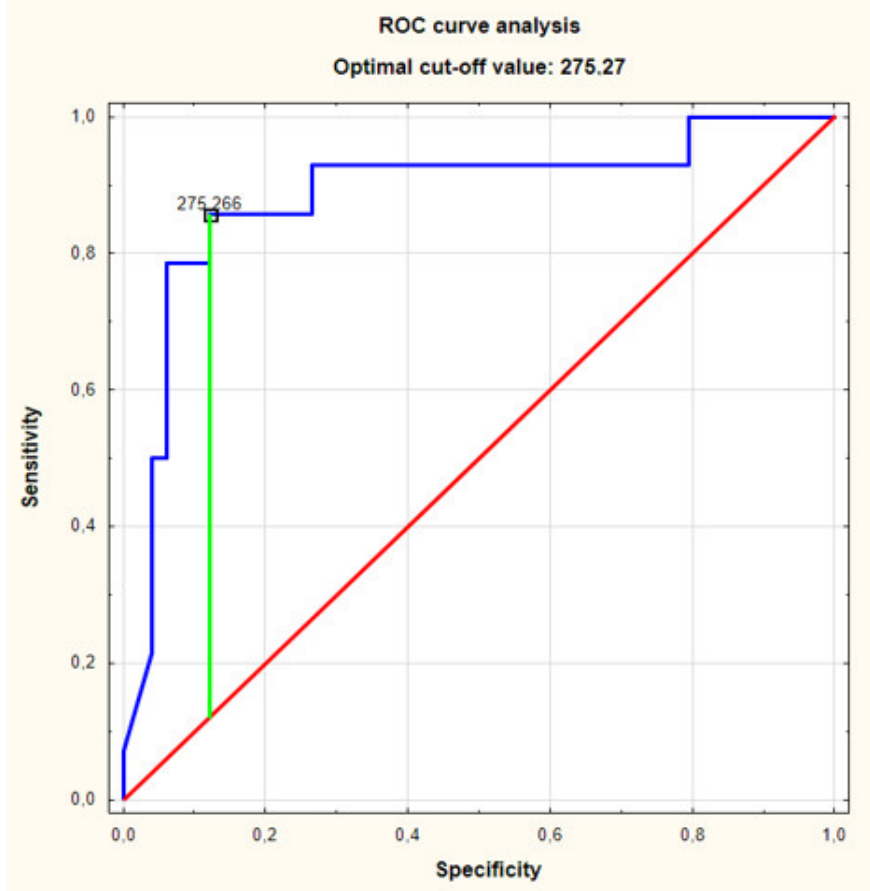

(c)

Figure 4. (a). ROC curve analsysis of relationship between serum concentrations of S100A15 in individuals with HS in stage Hurley III and controls showed area under the curve (AUC) of 0.81. (95\%CI for AUC: 0.66-0.97) $(p=0.0001)$. (b). ROC curves analysis of relationship between serum concentrations of S100A15 in individuals with HS in Hurley III stage and in individuals with HS in Hurley I stage revealed AUC of 0.95 (95\%CI for AUC: $0.86-1)(p<0.0001)$. (c). ROC curves analysis of relationship between serum concentrations of S100A15 in individuals with HS in Hurley III stage and in individuals with HS in less severe stages (Hurely I, Hurley II) revealed AUC of 0.88 (95\%CI for AUC: $0.77-0.99)(p<0.0001)$. 
The serum concentrations of S100A15 in the individuals with HS in Hurley stage III were significantly increased compared to those of the individuals with HS in Hurley stage I and the individuals with HS in Hurley stage II ( $p<0.0001$ and $p=0.0004$, respectively) (Figure $3 b$ ). The ROC curve analysis of the relationship between the serum concentration of S100A15 in the individuals with HS in Hurley stage III and in the individuals with HS in Hurley stage I revealed an AUC of 0.95 (95\%CI for AUC: 0.86-1) $(p<0.0001)$. The optimal cut-off value was $161.39 \mathrm{pg} / \mathrm{mL}$ (Figure $4 \mathrm{~b}$ ).

The ROC curve analysis of the relationship between the serum concentration of S100A15 in the individuals with HS in Hurley stage III and in the individuals with HS in the less severe stages (Hurley I and Hurley II) revealed an AUC of 0.88 (95\%CI for AUC: $0.77-0.99)(p<0.0001)$. The optimal cut-off value was $275.27 \mathrm{pg} / \mathrm{mL}$.

The serum concentration of S100A15 in the individuals with HS was positively correlated with their CRP concentration $(p=0.004, R=0.425)$ (Figure 5). We did not find any correlations between the serum concentrations of S100A15 and BMI, smoking habit or other demographic and clinical data.

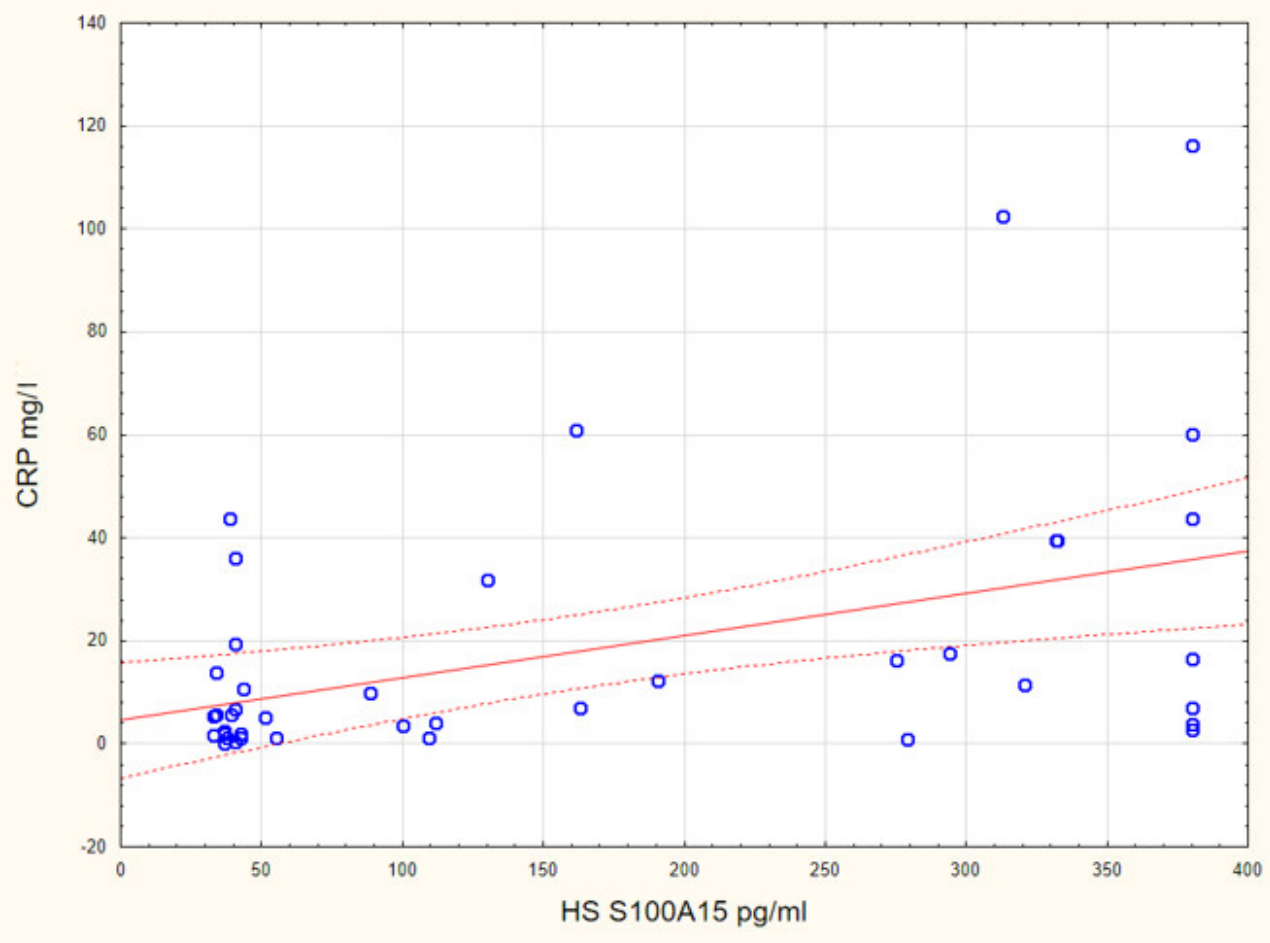

Figure 5. The serum concentration of S100A15 in individuals with HS was positively correlated with CRP concertation $(p=0.004, R=0.425)$.

\section{Discussion}

To the best of our knowledge, this is the first study to evaluate the serum levels of S100A4 and S100A15 in individuals with HS. We found significantly elevated serum S100A4 levels in the individuals with HS as compared to the controls. The highest serum level of S1004 was found in the patients with HS in Hurley stage II. S100A4 exerts proinflammatory action and has been shown to be involved in the pathogenesis of some chronic immunemediated inflammatory skin diseases, such as psoriasis and systemic sclerosis [16-20]. A weak and sparse expression of S100A4 was shown in the normal skin tested. Upon various proinflammatory stimuli, numerous inflammatory cells (e.g., lymphocytes, neutrophils and macrophages), fibroblasts and endothelial cells up-regulate the expression of S100A4 by releasing it into the extracellular space. S100A4 is an active extracellular factor with the capacity to influence gene expression by modulating numerous signaling pathways and transcription factors, including NF-кB. S100A4 also induces the expression 
of proinflammatory cytokines, such as IL-1 $\beta$, IL- 6 and TNF- $\alpha$, acute phase reactants and some other S100 protein family members (e.g., 100A8 and S100A9) which are known to be involved in the pathogenesis of HS $[1,11,16,25,26]$. Further, it promotes the recruitment and chemotaxis of inflammatory cells $[16,17,19-21]$. As a result of these known processes, S100A4 may enhance inflammatory process in HS. In the present study, we did not find any correlation between S100A4 serum level and CRP serum level. HS is associated with tissue remodeling [1], while S100A4 is considered to be a fibroblast marker, used to predict and monitor fibrosis in various tissue. In response to persistent inflammation, S100A4 induces tissue fibrosis and is involved in the transition of epithelial or endothelial cells into inflammation-induced fibroblasts. Further, it stimulates fibroblasts for the aberrant production of extracellular matrix (ECM) and induces the expression and activation of matrix metalloproteinases (MMPs) [16-18]. The course and progression of HS is hard to predict. All patients start in Hurley stage I and some of them progress to more advanced stages in which sinus tracts, fibrosis, scarring and dermal contractures occur $[1,2]$. Therefore, there is a need for the identification of biomarkers that may be useful in monitoring the progression of HS $[1,11]$. In the present study, the serum S100A4 levels in the individuals with HS in Hurley stages II and III were significantly increased compared to those in the individuals in Hurley stage I. The ROC curve analysis showed the AUC to be 0.72 . The optimal cut-off point was set at $6.16 \mathrm{pg} / \mathrm{mL}$. Therefore, we suggest that the serum level of S100A4 may serve as a biomarker to predict and monitor inflammatory processes and fibrosis in individuals with HS; in particular, S100A4 may facilitate differentiation between stage I and the more advanced stages according to Hurley classification. Further research is required to establish the role of S100A4 in the pathogenesis of HS and its usefulness as a serum marker in daily practice.

In the present study, we found elevated serum levels of S100A15 in the individuals with HS in Hurley stage III compared to those in the controls. The S100A15 serum levels in the individuals with HS were positively correlated with their CRP serum levels. S100A15 has a proinflammatory function and is known to potentiate inflammatory processes in the skin $[15,22-24]$. Furthermore, it has been shown to stimulate mononuclear cells circulating in peripheral blood, including lymphocytes and monocytes, for the increased expression and production of proinflammatory cytokines, such as TNF- $\alpha$, IL-1 $\beta$, IL-6 and IL-8 [27]. It also acts as a chemotactic factor for neutrophils and monocytes/macrophages [24]. TNF- $\alpha$, Il-1 $\beta$, IL- 6 and IL- 8 are involved both in the pathogenesis of HS as well as in the development of glucose intolerance, obesity, hyperlipidemia, metabolic syndrome and atherosclerosis $[1,3,4,9]$. Up-regulated by proinflammatory micromilieu, S100A15 may amplify both skin and systemic inflammation and contribute to the development of comorbidities in individuals with HS. Increased serum levels of S100A15 have also been found in patients suffering from psoriasis, and it has been recently proposed as a biomarker of subclinical atherosclerosis [28]. Recent studies have indicated that HS is linked with an increased risk of major adverse cardiac events (MACEs), which is even higher than the risk of MACE that is associated with psoriasis. Further, an increased frequency of subclinical atherosclerosis has been found in patients with HS [9,29]. In the present study, we found a positive correlation between the S100A15 serum level and the CRP level in the individuals with HS. It is suggested that the link between HS and increased cardiovascular risk results from chronic systemic inflammation $[9,29]$. The elevated level of CRP is considered to be a marker of inflammatory processes and an independent cardiovascular risk factor [30]. We did not find a correlation between S100A15 level and BMI or smoking habit. The observed serum level of S100A15 was significantly elevated in the individuals with HS in Hurley stage III compared to that in the individuals with HS in Hurley stage I. The ROC curve analysis revealed a large AUC of 0.95 . The optimal cut-off value was set at $161.39 \mathrm{pg} / \mathrm{mL}$. Therefore, the serum level of S100A15 may be suggested as a marker for the monitoring of the progression of HS. A limitation of this study is the small cohort of patients. The independent influences of smoking and BMI on the obtained results were not estimated. 
Further research is required to establish the relevance of S100A15 as a serum marker of systemic inflammation and subclinical atherosclerosis in individuals with HS.

Here, we suggest new serum biomarkers for the monitoring of HS progression. S100A4 and S100A15 may be involved in the pathogenesis of HS by promoting inflammatory processes and fibrosis.

Author Contributions: A.B.-B.-conception and design, analyzing data, preparing the manuscript. W.B.- analyzing data, preparing the manuscript. D.N.-S. - lab work, analyzing data. Ł.M.-collection of samples. J.C.S.- analyzing data, manuscript corrections. All authors have read and agreed to the published version of the manuscript.

Funding: This paper was funded by a Scientific Grants from Wroclaw Medical University. (SUB.C260.21.011 and STM.C260.16.028).

Institutional Review Board Statement: The study was conducted according to Declaration of Helsinki Principles and approved by the local ethical committee of Wroclaw Medical University (KB 520/2018).

Informed Consent Statement: Informed consent was obtained from all subjects involved in the study.

Data Availability Statement: All source data are available per request by email.

Conflicts of Interest: The authors have no conflicts of interest to declare.

\section{References}

1. Nguyen, T.V.; Damiani, G.; Orenstein, L.A.V.; Hamzavi, I.; Jemec, G.B. Hidradenitis suppurativa: An update on epidemiology, phenotypes, diagnosis, pathogenesis, comorbidities and quality of life. J. Eur. Acad. Dermatol. Venereol. 2021, 35, 50-61. [CrossRef]

2. Poli, F.; Jamec, G.B.; Revuz, J. Clinical presentation. In Hidradenitis Suppurativa; Jamec, G.B., Revuz, J., Leyden, J.J., Eds.; Springer: Berlin/Heidelberg, Germany, 2006; pp. 11-24.

3. Prens, E.; Deckers, I. Pathophysiology of hidradenitis suppurativa: An update. J. Am. Acad. Dermatol. 2015, 73, S8-S11. [CrossRef]

4. Kelly, G.; Sweeney, C.M.; Tobin, A.-M.; Kirby, B. Hidradenitis suppurativa: The role of immune dysregulation. Int. J. Dermatol. 2014, 53, 1186-1196. [CrossRef] [PubMed]

5. Lima, A.L.; Karl, I.; Giner, T.; Poppe, H.; Schmidt, M.; Presser, D.; Goebeler, M.; Bauer, B. Keratinocytes and neutrophils are important sources of pro-inflammatory molecules in hidradenitis suppurativa. Br. J. Dermatol. 2016, 174, 514-521. [CrossRef]

6. Scala, E.; Di Caprio, R.; Cacciapuoti, S.; Caiazzo, G.; Fusco, A.; Tortorella, E.; Fabbrocini, G.; Balato, A. A new T helper 17 cytokine in hidradenitis suppurativa: Antimicrobial and proinflammatory role of interleukin-26. Br. J. Dermatol. 2019, 181, 1038-1045. [CrossRef]

7. Scala, E.; Cacciapuoti, S.; Garzorz-Stark, N.; Megna, M.; Marasca, C.; Seiringer, P.; Volz, T.; Eyerich, K.; Fabbrocini, G. Hidradenitis Suppurativa: Where We Are and Where We Are Going. Cells 2021, 10, 2094. [CrossRef] [PubMed]

8. Dessinioti, C.; Katsambas, A.; Antoniou, C. Hidradenitis suppurrativa (acne inversa) as a systemic disease. Clin. Dermatol. 2014, 32, 397-408. [CrossRef]

9. Garg, A.; Malviya, N.; Strunk, A.; Wright, S.; Alavi, A.; Alhusayen, R.; Alikhan, A.; Daveluy, S.D.; Delorme, I.; Goldfarb, N.; et al Comorbidity screening in hidradenitis suppurativa: Evidence-based recommendations from the US and Canadian Hidradenitis Suppurativa Foundations. J. Am. Acad. Dermatol. 2021, 23, S0190. [CrossRef] [PubMed]

10. Skroza, N.; Mambrin, A.; Proietti, I.; Balduzzi, V.; Bernardini, N.; Marchesiello, A.; Michelini, S.; Tolino, E.; Volpe, S.; Maddalena, P.; et al. Evaluation of Cardiovascular Risk in Hidradenitis Suppurativa Patients Using Heart Rate Variability (HRV) Analysis. Cardiovasc. Ther. 2020, 2020, 1-7. [CrossRef]

11. Wieland, C.; Vogl, T.; Ordelman, A.; Vloedgraven, H.; Verwoolde, L.; Rensen, J.; Roth, J.; Boer, J.; Hessels, J. Myeloid marker S100A8/A9 and lymphocyte marker, soluble interleukin 2 receptor: Biomarkers of hidradenitis suppurativa disease activity? Br. J. Dermatol. 2013, 168, 1252-1258. [CrossRef]

12. Donato, R. Intracellular and extracellular roles of S100 proteins. Microsc. Res. Tech. 2003, 60, 540-551. [CrossRef] [PubMed]

13. Batycka-Baran, A.; Maj, J.; Wolf, R.; Szepietowski, J. The New Insight into the Role of Antimicrobial Proteins-Alarmins in the Immunopathogenesis of Psoriasis. J. Immunol. Res. 2014, 2014, 1-10. [CrossRef]

14. Cao, Y.; Hong, F.; Conlon, D.; Sidur, L.; Smith, K.; Fang, Y.; Cuff, C.; Kaymakcalan, Z.; Ruzek, M. Potential predictive biomarkers of adalimumab response in patients with hidradenitis suppurativa. Br. J. Dermatol. 2021, 185, 804-814. [CrossRef]

15. Wolf, R.; Ruzicka, T.; Yuspa, S.H. Novel S100A7 (psoriasin)/S100A15 (koebnerisin) subfamily: Highly homologous but distinct in regulation and function. Amino Acids 2010, 41, 789-796. [CrossRef]

16. Fei, F.; Qu, J.; Li, C.; Wang, X.; Li, Y.; Zhang, S. Role of metastasis-induced protein S100A4 in human non-tumor pathophysiologies. Cell Biosci. 2017, 7, 64. [CrossRef]

17. Li, Z.; Li, Y.; Liu, S.; Qin, Z. Extracellular S100A4 as a key player in fibrotic diseases. J. Cell. Mol. Med. 2020, 24, 5973-5983. [CrossRef] 
18. Tomcik, M.; Palumbo-Zerr, K.; Zerr, P.; Avouac, J.; Dees, C.; Sumova, B.; Distler, A.; Beyer, C.; Cerezo, L.A.; Becvar, R.; et al. S100A4 amplifies TGF-beta-induced fibroblast activation in systemic sclerosis. Ann. Rheum. Dis. 2015, 74, 1748-1755. [CrossRef]

19. Klingelhöfer, J.; Senolt, L.; Baslund, B.; Nielsen, G.H. Up-regulation of metastasis-promoting S100A4 (Mts-1) in rheumatoid ar-thritis: Putative involvement in the pathogenesis of rheumatoid arthritis. Arthritis Rheum. 2007, 56, 779-789. [CrossRef] [PubMed]

20. Zibert, J.R.; Skov, L.; Thyssen, J.P.; Jacobsen, G.K.; Grigorian, M. Significance of the S100A4 protein in psoriasis. J. Investig. Dermatol. 2010, 130, 150-160. [CrossRef]

21. Andrés Cerezo, L.; Kuncová, K.; Mann, H.; Tomčík, M.; Zámečník, J.; Lukanidin, E.; Neidhart, M.; Gay, S.; Grigorian, M.; Vencovský, J.; et al. The metastasis promoting protein S100A4 is increased in idiopathic inflammatory myopathies. Rheumatology 2011, 50, 1766-1772. [CrossRef] [PubMed]

22. Batycka-Baran, A.; Koziol-Galczynska, M.; Bieniek, A.; Wolf, R.; Łaczmański, Ł.; Szepietowski, J. Expression of koebnerisin (S100A15) and calgranulin A (S100A8) in lesional and perilesional skin in patients suffering from hidradenitis suppurativa. J. Eur. Acad. Dermatol. Venereol. 2020, 34, e402-e404. [CrossRef] [PubMed]

23. Hegyi, Z.; Zwicker, S.; Bureik, D.; Peric, M.; Koglin, S.; Batycka-Baran, A.; Prinz, J.C.; Ruzicka, T.; Schauber, J.; Wolf, R. Vitamin D Analog Calcipotriol Suppresses the Th17 Cytokine-Induced Proinflammatory S100 “Alarmins" Psoriasin (S100A7) and Koebnerisin (S100A15) in Psoriasis. J. Investig. Dermatol. 2012, 132, 1416-1424. [CrossRef]

24. Wolf, R.; Howard, O.M.Z.; Dong, H.-F.; Voscopoulos, C.; Boeshans, K.; Winston, J.; Divi, R.; Gunsior, M.; Goldsmith, P.; Ahvazi, B.; et al. Chemotactic Activity of S100A7 (Psoriasin) Is Mediated by the Receptor for Advanced Glycation End Products and Potentiates Inflammation with Highly Homologous but Functionally Distinct S100A15. J. Immunol. 2008, 181, 1499-1506. [CrossRef]

25. Jenei, A.; Dajnoki, Z.; Medgyesi, B.; Gáspár, K.; Béke, G.; Kinyó, Á.; Méhes, G.; Hendrik, Z.; Dinya, T.; Törőcsik, D.; et al. Apocrine Gland-Rich Skin Has a Non-Inflammatory IL-17-Related Immune Milieu, that Turns to Inflammatory IL-17-Mediated Disease in Hidradenitis Suppurativa. J. Investig. Dermatol. 2019, 139, 964-968. [CrossRef] [PubMed]

26. Frew, J.W.; Hawkes, J.E.; Krueger, J.G. A systematic review and critical evaluation of inflammatory cytokine associations in hidradenitis suppurativa. F1000Research 2018, 7, 1930. [CrossRef]

27. Batycka-Baran, A.; Hattinger, E.; Zwicker, S.; Summer, B.; Howard, O.Z.; Thomas, P.; Szepietowski, J.; Ruzicka, T.; Prinz, J.C.; Wolf, R. Leukocyte-derived koebnerisin (S100A15) and psoriasin (S100A7) are systemic mediators of inflammation in psoriasis. J. Dermatol. Sci. 2015, 79, 214-221. [CrossRef]

28. Awad, S.M.; Attallah, D.A.; Salama, R.H.; Mahran, A.M.; Abu El-Hamed, E. Serum levels of psoriasin (S100A7) and koebnerisin (S100A15) as potential markers of atherosclerosis in patients with psoriasis. Clin. Exp. Dermatol. 2018, 43, 262-267. [CrossRef]

29. González-López, M.A.; Hernández, J.L.; Lacalle, M.; Mata, C.; López-Escobar, M.; López-Mejías, R.; Portilla, V.; Fuentevilla, P.; Corrales, A.; González-Vela, M.C.; et al. Increased prevalence of subclinical atherosclerosis in patients with hidradenitis suppurativa (HS). J. Am. Acad. Dermatol. 2016, 75, 329-335. [CrossRef] [PubMed]

30. Lagrand, W.K.; Visser, C.A.; Hermens, W.T.; Niessen, H.W.; Verheugt, F.W.; Wolbink, G.J.; Hack, C.E. C-reactive protein as a cardiovascular risk factor: More than an epiphenomenon? Circulation 1999, 100, 96-102. [CrossRef] 